Pulsar Astronomy - 2000 and Beyond

ASP Conference Series, Vol. 202, 2000

M. Kramer, N. Wex, and R. Wielebinski, eds.

\title{
Possible re-acceleration regions above the inner gap and pulsar gamma-ray emission
}

\author{
B.H. Hong ${ }^{1,2,3}$, G.J. Qiao ${ }^{1,2}$, B. Zhang ${ }^{1,2}$, J.L. $\operatorname{Han}^{1,3}$, R.X. Xu ${ }^{1,2}$ \\ ${ }^{1}$ Beijing Astrophysical Center, CAS-PKU, Beijing 100871, China \\ ${ }^{2}$ Astronomy Department, Peking University, Beijing 100871, China \\ ${ }^{3}$ National Astronomical Observatories, CAS, Beijing 100080, China
}

\begin{abstract}
We propose that the violent breakdown of the RudermanSutherland type vacuum gap above the pulsar polar cap region may produce copious primary particles and make the charge density above the gap in excess of the Goldreich-Julian density. Such an excess residue of charge density may arise some re-acceleration regions above the gap due to the space-charge-limited flow mechanism. Such re-acceleration regions, if exist, may accelerate particles to carry away more spin-down energy, and may have some implications for pulsar $\gamma$-ray emission.
\end{abstract}

Much has been done to investigate the formation of pulsar inner accelerators near the polar cap region (Ruderman \& Sutherland 1975 [RS75]; Arons \& Scharlemann 1979; Harding \& Muslimov 1998) via various charge deficit mechanisms. Here we propose another possible particle acceleration mechanism in the pulsar inner magnetospheres via a charge excess mechanism. The violent breakdown of the vacuum gap (RS75) may produce extra charges to make the charge density above the gap deviated from the Goldreich-Julian (1969) density, so that the space-charge-limited flow mechanism may result in one or more re-acceleration regions above the gap.

Basic idea of the re-acceleration regions Above the gap, the secondaries do not contribute to the pure charge density, so the net contribution of $\rho$ is just the primary charge density. In the previous polar cap acceleration models (RS75; Arons \& Scharlemann 1979; Harding \& Muslimov 1998), it is assumed that $E_{\|}$is completely screened. However, a complete screening of the field requires both $E_{\|}=0$ and $\mathrm{d} E_{\|} / \mathrm{d} z=0$ to be achieved. This is only assumed in the previous models. In reality, both conditions can not be achieved simultaneously at the very beginning, and there must be some oscillation and relaxation processes to readjust $\rho$ and $E_{\|}$to achieve final screening. In principle, such a detailed process should be investigated explicitly. Here we present our first attempt to treat such a complicated process within the framework of the vacuum gap model (RS75). We argue that the charge density $\rho$ of the primary particles flowing out from the gap could be considerably in excess of $\rho_{\mathrm{gj}}$ if the breakdown is very violent, e.g. $\rho=\xi \rho_{\mathrm{gj}}$ with $\xi>1$. In the near surface regime (RS75), Poisson equation could be approximately treated as one-dimensional, i.e. $\mathrm{d} E_{\|} / \mathrm{d} z=4 \pi\left(\rho-\rho_{\mathrm{gj}}\right)$. Within the gap, since $\rho=0$, and $\rho_{\mathrm{gj}}>0$, the $E_{\|}$ gradient is negative, and $E_{\|} \simeq(2 \Omega B / c)(h-z)$ (RS75). Above the gap, the possible excess of the charge density $\left(\rho>\rho_{\mathrm{gj}}\right)$ actually changes the sign of the $E_{\|}$ 
gradient, so that $E_{\|}$starts to grow with the direction of $E_{\|}$unchanged. Thus another re-acceleration region is probably formed. Completely neglecting the screening fields of the pairs, our results show that a series of accelerators will be formed, each of which again limited by another pair formation front. At the top of each re-acceleration region, there is also an additional charge excess (denoted as $\xi_{2}, \xi_{3}$, etc.). The relation between the adjacent charge excess is approximately $\left(\xi_{3}-1\right) /\left(\xi_{2}-1\right)=1+h_{2} /\left(R+h_{1}\right)>1$, where $h_{1}, h_{2}$ are the heights of the first and second accelerators, and $R$ the stellar radius. We see that $\xi_{3}>\xi_{2}$, which means that the charge deviations could not be converged.

A complete treatment of the re-acceleration region must include the screening fields of the secondary pairs. Such fields are mainly determined by the number of the pairs, i.e., the multiplicity factor of the pairs with respect to the primaries (defined as $\eta$ ). It is possible that the re-acceleration fields could not be completely screened if $\xi$ is large enough, but $\eta$ is not large enough, and the structure of the re-accelerator is decided by the competition of $\xi$ and $\eta$. As mentioned above, more pair formation fronts will be formed above each reacceleration regions, so that the increase of $\eta$ is much faster than the increase of $\xi$. As a result, it is unlikely to form too many re-acceleration regions. Very probably, only one or two re-acceleration regions can exist. Detailed quantitative study of such re-acceleration regions is in progress.

About the luminosity of gamma-ray emission Observationally, $\dot{E}_{\text {rot }} / 4 \pi d^{2}$ is adopted as a parameter to select $\gamma$-ray pulsar candidates (Thompson 1996), and among the 8 known $\gamma$-ray pulsars, an empirical law of $L_{\gamma} \propto$ $\left(\dot{E}_{\text {rot }}\right)^{1 / 2}$ is found. Zhang \& Harding (1999) proposed a full polar cap cascade model based on the acceleration model of Harding \& Muslimov (1998), which can successfully interpret both the $\gamma$-ray and $X$-ray luminosities of the spinpowered pulsars. In principle, the full cascade picture can be also used in the vacuum gap models. However, with the possible existence of multipole fields near the surface (RS75), the energetics of the vacuum gap is usually not enough to interpret $L_{\gamma}$. If, however, one or more re-acceleration regions proposed here do exist above the gap, particles within these "quasi-gaps" can be also accelerated to highly relativistic energies to bring away more spin-down energy to $\gamma$-ray emission, which may interpret the observed $L_{\gamma}$ of pulsars.

\section{References}

Arons, J., \& Scharlemann, E.T. 1979, ApJ, 231, 854

Goldreich, P., \& Julian, W.H. 1969, ApJ, 157, 869

Harding, A.K., \& Muslimov, A.G. 1998, ApJ, 508, 328

Ruderman, M.A., Sutherland, P.G. 1975, ApJ, 196, 51 (RS75)

Thompson, D.J. 1996, in: Pulsars: Problems \& Progress, (eds.) S. Johnston, M.A. Walker, \& M. Bailes, ASP Conf. Ser. Vol. 105, p.307

Zhang, B., \& Harding, A.K. 1999, ApJ, in press 